\title{
EL ESTUDIO DEL MERCADO DE TRABAJO DE LOS JÓVENES EN LA OBRA DE LUIS TOHARIA
}

\author{
Cecilia Albert*, María Ángeles Davia ${ }^{\dagger}$ \\ *Universidad de Alcalá \\ †Universidad de Castilla - La Mancha
}

\section{Resumen}

Este artículo revisa la producción científica en torno al mercado de trabajo de los jóvenes en la que participó el profesor Luis Toharia Cortés. En él se revisan los estudios sobre la entrada de los jóvenes al mercado de trabajo, aquellos que estudian alguna característica del empleo de los jóvenes y, finalmente, los trabajos centrados en el empleo de los titulados universitarios y de formación profesional. El texto concluye con unas reflexiones finales que se derivan de las conclusiones de muchos de trabajos revisados acerca de los salarios de los jóvenes y sus condiciones laborales.

Palabras clave: Empleo de los jóvenes, educación, paro juvenil.

Clasificación JEL: J13, J21.

\begin{abstract}
This article surveys the scientific production around youth labour markets in which Professor Luis Toharia Cortés took part. We revise studies about youths entry in the labour market, about the main features of youth employment and about the labour market prospects of university and vocational training graduates. The article concludes with final reflexions stemming from the abovementioned research on wages for the young and their working conditions.
\end{abstract}

Key words: Youth employment, youth unemployment education.

JEL Classification: J13, J21.

\section{Introducción}

Uno de los aspectos que para Luis Toharia era fundamental y que debe sostener cualquier estudio sobre el mercado de trabajo es conocer cuántos somos, qué edad tenemos y qué sabemos hacer. Este planteamiento le llevó a prestar atención a todos los grupos demográficos y, entre ellos, a los jóvenes. Los jóvenes están presentes en la obra de Luis Toharia en la medida en la que la edad y el momento del ciclo vital en el que se encuentran las personas son variables clave para comprender el mercado laboral, pero también desarrolló trabajos en los que los jóvenes son el objeto primordial de análisis. Este último tipo de trabajos es, precisamente, el que se pretende recopilar y resumir aquí.

(c) Revista de Economía Laboral 
En los últimos 30 años ha habido una constante preocupación en la sociedad española por la elevada tasa de paro juvenil y las dificultades de los jóvenes para encontrar un "buen" trabajo. Estas dificultades se dan en un contexto histórico de aumento sostenido del nivel educativo de los jóvenes, lo que ha llevado en muchas ocasiones a establecer relaciones de causalidad entre ambos fenómenos: expansión educativa y deterioro de los indicadores laborales de los jóvenes. Pero las cosas no son tan sencillas; de hecho, aunque se trata de un tema en el que se lleva trabajando desde mediados de los años ochenta del siglo XX, no se conocen bien todavía qué factores favorecen (o perjudican) a los jóvenes a la hora de relacionarse con el mercado de trabajo. Esta pregunta se concreta de diferente manera en cada uno de los trabajos de Luis Toharia que vamos a recoger aquí, como por ejemplo, ¿Es el salario de los jóvenes demasiado alto? ¿Influye el ciclo económico en el logro de un empleo? ¿Ayuda el nivel educativo a la hora de encontrar un trabajo?, etc. Luis Toharia abordó todas estas preguntas con gran rigor metodológico, condicionado a los datos disponibles en cada momento.

Esta revisión de una pequeña parte de obra de Luis Toharia se estructura en tres apartados, además de esta introducción. En el apartado 2 se revisan los estudios más relacionados con la entrada de los jóvenes al mercado de trabajo. El tercer apartado está dedicado a los trabajos que estudian alguna característica del empleo de los jóvenes. En el cuarto apartado se repasan los trabajos centrados en el empleo de los titulados universitarios y de formación profesional. La exposición termina con un apartado de reflexiones finales.

\section{La entrada de los jóvenes al mercado de trabajo}

Esta sección recoge una serie de trabajos que comparten un núcleo común y, al mismo tiempo, son muy diversos. Lo que tienen en común es el interés por conocer las decisiones de los jóvenes en lo que respecta a su inversión en educación y las transiciones desde el sistema educativo, y muy en especial la entrada en el mercado de trabajo: tiempos, condiciones y calidad de los primeros empleos, entre otros asuntos. Las investigaciones que aparecen resumidas aquí tienen también en común que, desde el punto de vista de la metodología, se adaptan perfectamente a la información disponible en cada momento y a las técnicas más adecuadas también al alcance de los investigadores. Por ejemplo, desde los años noventa se hizo uso de las potencialidades que ofrecía los ficheros longitudinales de la Encuesta de Población Activa (EPA) y del Panel de Hogares de la Unión Europea (PHOGUE), siempre con cautela por las 
limitaciones de las fuentes. En la década siguiente se exploraron los resultados que ofrecía la Encuesta de Transición Educativo-Formativa e Inserción Laboral (ETEFIL) y la Muestra Continua de Vidas Laborales (MCVL). Cada una de ellas ofrece matices diferentes, desde la amplia representatividad de la EPA y la MCVL a las posibilidades de comparaciones internacionales a través del PHOGUE, pasando por el estudio de los jóvenes que abandonan tempranamente el sistema educativo (ETEFIL).

El trabajo "Choosing between education, training and labour market entry" (Albert et al, 2002) explora las decisiones de los jóvenes de estudiar o incorporarse al mercado de trabajo. Estas decisiones se analizan para tres niveles educativos: educación obligatoria, superior y formación profesional. Una de las aportaciones del trabajo es que compara tres países europeos: Alemania, Irlanda y España, lo cual fue posible gracias a la aparición del PHOGUE. En el año en que se realizó el trabajo sólo estaba disponible la primera ola (correspondiente a 1994) y no se pudo incorporar un estudio de transiciones, de modo que únicamente se aborda un análisis de corte trasversal. El trabajo forma parte de una investigación mucho más amplia en el marco de un proyecto europeo que tenía como objetivo aportar evidencia empírica para el contraste de la corriente de pensamiento liderada por Günther Schmid (entonces investigador del WZB (Wissenschaftszentrum Berlin für Sozialforschung, Alemania) llamada "Mercados de trabajo transicionales". Los resultados obtenidos en este trabajo no estaban en consonancia con las predicciones de la teoría neoclásica del capital humano, especialmente en las variables que miden la influencia de las expectativas de empleo y renta sobre la decisión de estudiar, lo que invitaba a reflexionar sobre la posible influencia de las instituciones en el comportamiento de los jóvenes, como por ejemplo, las diferencias entre países en los modelos prevalentes de familia o en la oferta educativa. Hay un gran consenso en que los aspectos institucionales influyen en el comportamiento de las personas, pero también, al mismo tiempo, serias dificultades metodológicas a la hora de cuantificar o determinar el efecto neto de las instituciones, pues difícilmente se pueden incorporar en los modelos al uso.

La disponibilidad de los ficheros longitudinales de la EPA permite observar a los jóvenes en (al menos) dos momentos diferentes del tiempo y supone un avance, para el caso de España, respecto a trabajos anteriores (como el que se acaba de comentar), en la medida en la que permite estudiar transiciones del sistema educativo al mercado de trabajo. El análisis de transiciones representa, en los años noventa, un reto para la investigación de los determinantes de la salida del sistema educativo reglado y la entrada al mercado de trabajo. Luis Toharia, en colaboración 
con otros autores, desarrolla varios trabajos que tienen como objetivo estudiar la transición de la escuela al mercado de trabajo con los ficheros longitudinales de la EPA. El primer trabajo fue publicado en 1997 por el IVIE y lleva por título "La transición de los jóvenes de la escuela al mercado de trabajo: un análisis longitudinal" (Albert, Juárez y Sánchez, 1997). Este es un trabajo fundamentalmente metodológico, en el que se exploran las potencialidades y limitaciones de la base de datos para el estudio de las transiciones de los jóvenes. La primera cuestión que se plantea es la necesidad de definir con claridad cada una de las situaciones en las que puede encontrarse un joven: estudiante, inactivo, ocupado y parado. Lo que en principio parece bastante claro en la vida cotidiana, al tratar de trasladarlo a la información estadística disponible en la encuesta resulta complejo. La razón es que las actividades educativas y la situación laboral son perfectamente compatibles, lo que lleva a la necesidad de buscar una jerarquía entre ellas, de manera que las definiciones estadísticas se ajusten lo más posible a la realidad y además sean operativas para el análisis.

El trabajo "La transición de la escuela al mercado de trabajo: años noventa" (Albert, Juárez y Sánchez, 2000) aprovecha la incorporación de nuevas olas de la EPA enlazada para analizar la influencia del ciclo económico sobre los flujos del sistema educativo al mercado de trabajo, es decir, la salida del sistema educativo y la posterior situación laboral del joven (empleo, paro o inactividad). El análisis abarca el periodo 1992-1999, que contiene un estadio de fuerte recesión (19921995) y otro de leve recuperación (1995-1999). Esto permite preguntarse hasta qué punto la mejor situación del mercado de trabajo que se observa en España en los últimos años de la década de 1990 ha facilitado las salidas "virtuosas" del mercado de trabajo (es decir, las salidas hacia el empleo), como cabría esperar a priori. La respuesta que se encontró en este trabajo a esta pregunta es que el ciclo económico afectó sobre todo a las decisiones educativas de los varones, y muy poco a las de las mujeres. La mejoría de la situación económica llevó a los varones a abandonar con mayor frecuencia sus estudios, impulsados por las mayores oportunidades de empleo. En el caso de las mujeres, sin embargo, debido seguramente a la menor tendencia cíclica del empleo femenino, no se observaba una mayor probabilidad de encontrar empleo a pesar de la mejora en las condiciones económicas generales. Además, seguramente las mujeres consideraban que invertir en capital humano es más importante para su futura carrera laboral en mayor medida que los varones, por lo que mantenían una actitud más centrada en los estudios y menos atenta a las posibles oportunidades de empleo. En el artículo también se analiza la influencia de las características personales (sexo, edad y estudios) y 
familiares (nivel educativo, condición socioeconómica y situación laboral del padre y/o la madre), y llega a la conclusión de que estas influyen en la transición del sistema educativo al mercado de trabajo, aunque con relevantes diferencias entre los varones y las mujeres.

"Del sistema educativo al mercado de trabajo: un análisis de flujos" (Albert, Juárez y Sánchez, 2003) es un trabajo donde se analizan las diferencias específicas entre los distintos niveles educativos en la salida al mercado de trabajo, lo que permitió matizar algunos de los resultados de trabajos anteriores. En primer lugar, confirmó que los varones tienen una mayor probabilidad de salida del sistema educativo que las mujeres y, una vez que han abandonado el sistema educativo, presentan mayor probabilidad de estar ocupados y menor de realizar una transición al desempleo. Por otra parte, las mujeres que no han conseguido el título de estudios obligatorios presentan mayores salidas a la inactividad que las más cualificadas, mientras que las mujeres con estudios universitarios presentan menores transiciones a la inactividad. Por último, son más frecuentes las salidas al desempleo en varones y mujeres universitarias que han conseguido la titulación, mientras que las salidas más frecuentes a la ocupación se producen tanto para estudiantes universitarios de ambos sexos que no han conseguido la titulación. En realidad, en este último caso, el abandono de la titulación viene influenciado por haber encontrado un empleo; lo que el estudio no aborda es qué condiciones laborales se logran en ese empleo o la calidad del empleo que alcanzan estos jóvenes.

El uso de la EPA enlazada para el análisis de transiciones representó en su momento un gran avance respecto a los estudios de corte trasversal, pero el hecho de no ser un panel "puro" exigió un especial cuidado en la definición de los flujos y en la interpretación de los resultados. Del mismo modo, una vez que los datos mostraron que los flujos entre la escuela y el mercado de trabajo iban en todas direcciones y además, tal y como era de esperar, participar en el sistema educativo era compatible con cualquier situación laboral (o viceversa), fue necesario definir con claridad qué condiciones debía cumplir un entrevistado para ser considerado como estudiante.

Estos matices fueron incorporados en las estaídticas cuando, a mediados de la década pasada, se hizo pública una base de datos diseñada exclusivamente para estudiar la inserción laboral de los egresados en estudios no universitarios: la Encuesta de Transición EducativoFormativa e Inserción Laboral ETEFIL, un proyecto común de los Ministerios de Educación y Ciencia, de Trabajo y Asuntos Sociales y el Instituto Nacional de Estadística, que consta de siete encuestas dirigidas a diferentes colectivos de jóvenes no universitarios: entre ellos se 
encuentran los jóvenes que en el año 2001 tenían menos de 25 años y habían en ese año abandonado el Graduado en Educación Secundaria Obligatoria (ESO), o bien obtuvieron el Graduado en ESO, o el Bachillerato, lograron Ciclos de Formación Profesional (FP) de Grado Medio o Ciclos de Grado Superior así como jóvenes que habían finalizado un curso del Plan de Inserción Profesional (FIP) o estudios en Escuelas Taller y Casas de Oficio (ETCO).

Con el objeto de afrontar un estudio exhaustivo de la educación secundaria, que ha recibido mucha menos atención en la literatura que la educación superior, en "La transición de la educación secundaria (obligatoria y no obligatoria) al mercado laboral" (Albert, Davia y Toharia, 2009) se analizaron las diferencias en la transición al mercado de trabajo entre los que abandonan los estudios de la ESO, los que obtienen el título de ESO y los que obtienen el de Bachillerato. También comparan resultados laborales iniciales de los estudiantes de los ciclos de FP de Grado Medio. Diferencian en los estudios generalistas (ESO y Bachillerato) a quienes terminan su título a la edad esperada y aquellos que lo obtienen más tarde. El estudio adopta además una perspectiva longitudinal, al considerar un periodo de 36 meses contados a partir del momento en el que los jóvenes alcanzaron los estudios o abandonaron la ESO. En ese periodo se analiza tanto el tránsito a su primer empleo "significativo" como las características del mismo. Los resultados más relevantes muestran que los estudiantes que no sufren retrasos en sus estudios de secundaria son los que tienen más probabilidades de demandar estudios superiores en el futuro. Dicho de otro modo, los problemas de rendimiento (o de cualquier otro tipo) experimentados en la secundaria, especialmente en la etapa obligatoria, tienen consecuencias serias y duraderas en la trayectoria educativa de los jóvenes. Otros resultados del trabajo muestran que la formación general que aporta la ESO y el Bachillerato no presenta inicialmente ventajas relevantes de cara al tiempo necesario para lograr un primer empleo "significativo" (al menos 20 horas de trabajo a la semana y 6 meses de duración) si los comparamos con los que abandonaron la ESO, cosa que sí ocurre con los Ciclos de FP de Grado Medio, mucho más orientados al mercado de trabajo. Por otro lado, se advierten ciertas ventajas de haber alcanzado un título de educación post obligatoria en las condiciones de los empleos a los que acceden los jóvenes no universitarios.

Este trabajo forma parte de un proyecto cuyos resultados aparecen de forma más exhaustiva en el informe de investigación "Exclusión social y pobreza: transición educativo - formativa e inserción laboral en la población joven" (Albert, Davia y Toharia, 2008), resultado de un Proyecto de investigación financiado por el programa 
FIPROS de la Seguridad Social, donde se prestaba especial atención al abandono escolar temprano en España, es decir, el abandono escolar que se produce tras la ESO. España presenta uno de los mayores niveles de los países de nuestro entorno en fracaso escolar, y resultaba de gran interés avanzar cuáles eran los resultados laborales de los jóvenes menos cualificados, dicho de otra manera, cuál es el coste que debían pagar en el mercado de trabajo por su escasa cualificación inicial. En el ámbito de los jóvenes poco cualificados se distinguieron las situaciones claras de fracaso escolar de las de retraso en el logro del Graduado en ESO y se identificaron distintos indicadores de riesgo de exclusión educativa, que se describieron en función de características socio-demográficas. La evaluación de las consecuencias de los distintos logros educativos en los niveles de educación no universitaria se abordó con una descripción de las trayectorias educativas y laborales de estos jóvenes, su paro por experiencias de inactividad y desempleo tras finalizar e incluso abandonar los estudios y el logro de "empleos significativos", así como las características de los mismos.

En una muestra más de la versatilidad para afrontar el estudio de la entrada en el mercado de trabajo a través de fuentes administrativas y secundarias, en "La entrada en el mercado de trabajo. Un análisis basado en la Muestra Continua de Vidas Laborales" (Cebrián y Toharia, 2008) se aprovechó el potencial de la Muestra Continua de Vidas Laborales (MCVL) correspondiente a los años 2004, 2005 y 2006, para detectar la primera entrada en el mercado durante un periodo largo del tiempo y las condiciones en que se produce esta entrada ${ }^{1}$. Tras estudiar la probabilidad de acceder al mercado de trabajo mediante un contrato temporal o un contrato indefinido, se abordan las diferencias que hay en las trayectorias laborales que siguen quienes entran en el mercado con cada tipo de contrato. Entre las principales conclusiones de ese trabajo cabe destacar que los contratos indefinidos son una vía de entrada en el mercado de trabajo preferentemente para los trabajadores más cualificados, que la cualificación es la característica que más incide en el tiempo necesario para transitar desde un contrato temporal inicial a uno indefinido y en el nivel de permanencia en el empleo de quienes empiezan con un contrato indefinido su carrera laboral. También se observa que los trabajadores que acceden al mercado de trabajo con contratos de obra o

\footnotetext{
${ }^{1}$ La MCVL es una muestra aleatoria pura que contiene el 4 por ciento de las personas que tuvieron alguna relación con la Seguridad Social, como cotizantes o como beneficiarios de pensiones o prestaciones por desempleo, en este caso en los años 2004, 2005 y 2006, si bien ha venido publicándose con carácter anual desde entonces. No necesariamente se trata de jóvenes, pero sí de nuevos entrantes en el mercado de trabajo, de los cuales, la gran mayoría son jóvenes.
} 
servicio necesitan más tiempo para conseguir un empleo indefinido que los demás. En cambio, no se observan diferencias significativas entre los contratos indefinidos ordinarios y los de fomento del empleo en términos de permanencia en el primer empleo que se registra en la Seguridad Social.

\section{El empleo de los jóvenes}

El estudio del mercado de trabajo de los jóvenes abarca mucho más que el mero análisis de las transiciones desde el sistema educativo. Entre otras cosas, contempla cuestiones acerca de la calidad del empleo, dos de cuyos indicadores principales son el salario y el tipo de contrato. También la permanencia en el empleo puede ser un indicador de su calidad. Además, una vez en el mercado de trabajo los jóvenes se mueven entre empleos y entre estados laborales. En esta sección recogemos trabajos que estudian todos estos aspectos. Al igual que en la sección anterior, en esta aparecen investigaciones que aprovechan en cada momento la información más relevante disponible y la abordan con las técnicas más adecuadas.

El salario es una de las características más importantes a la hora de estudiar el empleo. “Es elevado el salario de los jóvenes?” (Muro y Toharia, 1989) es la pregunta que se plantea en este artículo publicado en 1985 en la Revista de Economía y Sociología del trabajo. Con la Encuesta de Condiciones de Vida y Trabajo de 1985 los autores estudian la probabilidad de que una persona gane más de 50 mil de las antiguas pesetas al mes, que serían aproximadamente 840 euros de hoy (una vez actualizada la capacidad adquisitiva de la renta a través del IPC). La teoría económica afirma que los salarios son resultado de la productividad de los trabajadores, que no es una característica observable de estos. Los autores creen que las diferencias salariales entre jóvenes y adultos son, en parte, reflejo de diferencias de productividad entre ambos tipos de trabajadores, pero han de afrontar esta afirmación con prudencia e invitan a la reflexión: frente a aquellos que se apresuran a adoptar medidas que pretendan reducir el salario de los jóvenes para hacer reflejar mejor la productividad de los jóvenes y hacer así frente al problema del paro juvenil, los autores subrayan que es importante determinar si las diferencias salariales observadas entre trabajadores de distintas edades reflejan o no diferencias de productividad.

En "Flexibilidad, juventud y trayectorias laborales en el mercado de trabajo español" (Toharia, Davia y Hernanz, 2001) se atiende a cuestiones que generalmente no aparecen en los registros administrativos como los del antiguo Instituto Nacional de Empleo 
(INEM) o encuestas como la EPA y el PHOGUE, puesto que se trata del resultado de un proyecto del Centro de Investigaciones Sociológicas (CIS) donde se explotó la encuesta 2321 "Opiniones y actitudes de los españoles ante el mercado de trabajo" de 1999. El estudio contribuyó a confirmar que la especial vulnerabilidad de los jóvenes ante las crisis económicas en España les ha llevado a refugiarse en el sistema educativo y en la familia, de manera que la entrada al mercado de trabajo es un proceso que se extiende cada vez más en el tiempo. Pero quizá una de las conclusiones más relevantes del estudio es el impacto que la llamada "cultura de la temporalidad" ha tenido en el empleo de la juventud: esta se identifica sobre todo con el empleo juvenil inestable y con trayectorias caóticas al inicio de la vida laboral, que podrían condicionar el éxito laboral a lo largo de la vida.

Las estadísticas laborales europeas se han hecho eco de la existencia de empleo inestable a través del concepto de lo que Eurostat (la oficina estadística de la Unión Europea) llama empleo "significativo". Como ya hemos comentado anteriormente, el empleo "significativo" es definido por las estadísticas europeas y, por tanto, por las españolas, como el primer empleo con más de 20 horas de trabajo a la semana y un contrato de 6 o más meses de duración. Lograr un empleo con estas características puede ser considerado como una entrada de cierta "calidad" en el mercado de trabajo. El Módulo de la EPA del año 2000 (Transición de la educación al mercado laboral) permite diferenciar este primer empleo significativo de otras circunstancias laborales y medir bastante mejor que antes la calidad de la entrada en el mercado de trabajo. Su aparición permitió realizar tres estudios complementarios: en primer lugar, un análisis de los factores que influyen en el tiempo que los jóvenes tardan en encontrar un primer empleo significativo ("The Process of Finding the First "Good" Job: The Case of Spain" (Albert, Davia y Toharia, 2003); en segundo lugar, en la medida en que el módulo del año 2000 era un proyecto europeo, la existencia de módulos equivalentes en otros países permitió una comparación entre España e Italia de los factores que afectan a la probabilidad de que los jóvenes encuentren este empleo significativo ("Finding the first 'significant' job in Italy and Spain" (Albert, Davia y Toharia, 2007); y por último, se abordó el análisis de dos tipos de empleo, significativo y no significativo, frente a la posibilidad de no haber encontrado ningún empleo ("To Find or not to Find a Significant Job: The Case of Spain" (Albert, Toharia y Davia, 2008).

Estos tres trabajos analizan la década de los noventa y llegan a tres resultados fundamentales. En primer lugar, el mercado de trabajo español se caracteriza por un aumento de la cualificación de los jóvenes que al mismo tiempo tienen serias dificultades para encontrar un empleo 
de "calidad", lo cual es mucho más patente en las mujeres que en los varones. En segundo lugar, tanto en España como en Italia, los estudios universitarios y la formación profesional de grado superior ofrecen ventajas frente al resto de niveles educativos a la hora de encontrar un empleo significativo, resultado que no debe llevarnos a pensar que la calidad del empleo sólo depende de las características de la oferta de trabajo, ya que la demanda juega un papel primordial que en estos análisis no es por lo general tenido en cuenta. Y en tercer lugar, el campo o la rama de estudios tiene relevancia a la hora de encontrar un primer empleo significativo tanto en España como en Italia.

\section{El mercado de trabajo de los titulados (datos administrativos)}

Los datos de la EPA enlazada no sólo permiten estudiar transiciones en el sistema educativo y en el mercado de trabajo; también posibilitan estudiar las "no transiciones", es decir, la permanencia de los jóvenes en una situación concreta. El artículo "El abandono o la persistencia en los estudios universitarios" (Albert y Toharia, 2000) explora los determinantes de que los jóvenes sigan estudiando en la universidad y no la abandonen, ya sea para incorporarse al mercado de trabajo o a la inactividad. En este artículo (de manera deliberada) se rehúye del concepto de "fracaso universitario" debido a que se considera que la universidad es un nivel no obligatorio que los jóvenes pueden abandonar sin que necesariamente esto sea un fracaso de los jóvenes ni de las instituciones universitarias. Esta idea se basa en el convencimiento de que los estudios mejoran la empleabilidad de los jóvenes, incluso cuando estos no han alcanzado el título. Es decir, el rendimiento de la educación debe de encontrarse en algún punto intermedio entre la teoría del capital humano y la teoría credencialista, o dicho de otro modo, "el título importa" pero no lo es todo. Estas Ideas parecen haber sido relegadas al olvido hoy en día en la medida en que el abandono de los estudios universitarios por parte de los jóvenes se considera un fracaso y un mal uso de los recursos educativos públicos, lo que lleva a plantear políticas educativas centradas en la retención del estudiante en el centro hasta que alcance un título.

En este trabajo destacan tres resultados y de él se desprende una reflexión muy relevante. El primer resultado es que el área de conocimiento en la que se encuentra la titulación que cursan los jóvenes es 
importante en la probabilidad de abandonar los estudios universitarios ${ }^{2}$. El segundo resultado es que el logro de un empleo y la mera búsqueda activa de empleo (en el contexto de estudiantes a tiempo completo estos jóvenes son identificados en la EPA como desempleados) hacen aumentar de forma importante el abandono universitario. Esto puede tener varias lecturas: una cierta incompatibilidad entre los estudios y el mundo del trabajo, la utilización de los estudios universitarios como un "mecanismo de espera" mientras se encuentra trabajo o bien restricciones presupuestarias que hacen inviable la continuación de los estudios y obligan a los jóvenes a trabajar o, al menos, a buscar un empleo. La reflexión final del artículo es que es necesario conocer la trayectoria laboral de tres tipos de personas: los que tienen éxito, los que acaban con retraso y los que abandonan, una vez que han salido de la universidad. Debe evitarse que cualquier debate sobre la función social de la universidad, y en concreto sobre posibles reformas universitarias, se establezca en un contexto de especulaciones sobre las causas de los problemas suscitados. Únicamente un esfuerzo en la investigación profunda de los hechos, basada en información suficiente sobre los mismos, permitirá que las diversas posturas converjan hacia la mejor solución para todos, o al menos la más satisfactoria.

La falta de información para poder comprender las decisiones educativas y sus consecuencias para las experiencias laborales ha llevado a las universidades a desarrollar estudios particulares sobre la inserción laboral de sus titulados, de manera que, en la actualidad, prácticamente todas las universidades españolas realizan estudios de inserción laboral. Estos estudios se sustentan sobre la idea de que los logros laborales pueden ser una buena medida de la calidad de la enseñanza y de la universidad en su conjunto. Esta idea, aunque generalmente aceptada, no está exenta de matices. No parece razonable atribuir todo el mérito de la inserción laboral a la universidad y olvidarse de algunos elementos que no sólo pueden favorecer la inserción laboral sino también la propia labor de enseñanza de la universidad. Citaremos algunos de estos aspectos: la procedencia socioeconómica de los alumnos puede influir en su rendimiento académico y en sus aspiraciones laborales; el entorno socioeconómico de la universidad puede facilitar las relaciones de esta con

2 Desafortunadamente no se disponía en aquel momento de información suficiente para contrastar si esto se debía al grado de dificultad de la carrera, a las notas de corte de cada titulación que pueden favorecer una distribución desigual del alumnado en términos de preparación o a la motivación de los alumnos como consecuencia del lugar que ocupa la carrera que cursan en sus preferencias, es decir, hasta qué punto los estudiantes se encuentran cursando los estudios que preferían en primer lugar cuando resolicitaron el acceso en la universidad. 
el mundo del trabajo; y la selección de los mejores estudiantes, realizada no solo a través de la nota de entrada sino también a través de los requisitos administrativos exigidos para que estos se mantengan en la titulación una vez iniciada.

$\mathrm{Ni}$ siquiera disponer de información sobre todos estos elementos sería suficiente para mejorar nuestra percepción acerca de la calidad de nuestras universidades. Para avanzar en este camino sería imprescindible realizar estudios comparativos de las titulaciones entre distintas universidades. Aunque parece que hay un alto nivel de consenso en torno a esta idea, hasta la fecha no se han creado las condiciones de colaboración entre universidades que permitan desarrollar estos análisis. Quizás el miedo a desvelar nuestros defectos a los competidores es la causa de que no se agilice la realización de este tipo de estudios.

Por otro lado, los estudios particulares que han hecho un gran número de universidades hasta el momento sobre la inserción laboral de sus titulados suelen vincular a una o pocas titulaciones, lo que todavía "atomiza" más la evidencia existente en España sobre la inserción laboral de los egresados universitarios. Quizá uno de los mayores problemas de este tipo de estudios es que normalmente conllevan la realización de una encuesta que consume importantes recursos de la universidad y que además, al no seguir criterios homogéneos todas las universidades, no permite comparar los resultados con los de otros estudios o encuestas. Una solución a este problema consistiría en aprovechar la información de los registros administrativos procedente de la gestión académica de las universidades y enlazarlos con los ficheros del actual Servicio Público de Empleo Estatal, de la Seguridad Social y de la Agencia Tributaria. El potencial de una base de datos de este tipo a la hora de generar indicadores homogéneos de inserción laboral es importantísimo y el coste económico muy reducido. Sin embargo, la labor de coordinar a diversas administraciones públicas, a día de hoy todavía no se ha conseguido.

Con la idea que se expone en los párrafos anteriores se diseñó el estudio "La inserción laboral de los universitarios: un estudio piloto de los licenciados en Economía y Administración y Dirección de Empresas de la Universidad de Alcalá" (Albert, García-Serrano y Toharia, 2008) donde se abordó la inserción laboral de los licenciados en Economía y en Administración y Dirección de Empresas (ADE) de la Facultad de Ciencias Económicas y Empresariales de la Universidad de Alcalá pertenecientes a las promociones de 19981999 a 2002-2003. La elección de varias promociones obedece al intento de obtener una visión lo más amplia posible en el tiempo del proceso de incorporación al mercado de trabajo y de los cambios que se operan en los empleos que ocupan los titulados. Debe aclararse que aquí se utiliza el 
término promoción para denominar al grupo de individuos que han obtenido el título en el mismo año. Se contrapone, por tanto, la promoción de salida a la promoción de entrada, que denotaría a los alumnos que comienzan a estudiar a la vez en un momento dado. Si bien examinar el proceso de evolución en el tiempo de los alumnos que empiezan unos estudios determinados tiene su interés (sobre todo, para entender la dinámica del abandono y la duración de los estudios), los trabajos que analizan la inserción laboral de los titulados se centran en el análisis de quienes terminan los estudios a la vez, lo que homogeneiza a los individuos en un sentido: todos ellos se exponen al mercado laboral con los mismos credenciales (al menos, con el mismo título universitario) en el mismo momento del tiempo.

En este trabajo se construyó una base de datos con información que procedía de los servicios de Gestión Académica de la Universidad de Alcalá que se enlazó con los datos obtenidos de una encuesta a los titulados realizada a principios del año 2005. Esto permitió conectar características socio-demográficas y académicas con diversos aspectos relativos a su carrera laboral: su situación laboral actual, los rasgos de su inserción en el mercado de trabajo, su posterior progresión mediante la comparación entre las características de su primer empleo y de su último empleo, el grado de ajuste de los puestos de trabajo ocupados por los licenciados con la titulación cursada y las competencias para el desempeño del trabajo.

En otro trabajo relacionado con el anterior, "Los registros administrativos y los estudios de inserción laboral de los titulados universitarios: el caso de los titulados en Economía y ADE de la Universidad de Alcalá" (Albert, García-Serrano y Toharia, 2007) se da un paso más al unir a las dos bases de datos anteriores los datos procedentes del Servicio Público de Empleo Estatal, lo que permitió diseñar una serie de indicadores de inserción laboral que podrían ser obtenidos para cada una de las universidades españolas, y dentro de estas, para cada titulación, y además, para diversas características de los jóvenes o de las universidades en las que estos hayan estudiado.

Como el lector habrá advertido ya, estos trabajos pretendían diferenciarse de otros previos y aportar valor añadido al análisis de la inserción laboral de los titulados debido a la utilización de información suplementaria a la extraída a partir de encuestas a titulados. Se trataba de aprovechar la información administrativa de los titulados. Con estos antecedentes, el informe "Estudio de la Situación Laboral de las personas egresadas en enseñanzas universitarias y de Formación Profesional reglada en Andalucía: año académico 2005-2006" (Albert, Toharia y Villalcorba, 2008) muestra la potencialidad informativa y de análisis que ofrecería cruzar bases de datos procedente de diferentes 
administraciones en España. En este estudio se analiza la situación laboral de los graduados en ciclos formativos de FP y de egresados universitarias que se graduaron en cualquier universidad de Andalucía en el curso 2005-2006. Para ello utiliza una base de datos que procede del cruce de ficheros de gestión académica de los Institutos de educación secundaria y las universidades con los datos del Observatorio ARGOS del Servicio Andaluz de Empleo, que a su vez proceden de ficheros del Servicio Público de Empleo Estatal (demandas, colocaciones y contratos) y de los de la Seguridad Social. Este estudio fue un primer intento de explorar la información procedente de distintas fuentes registrales y proporcionar así una idea de la potencialidad de este tipo de datos, en especial cuando se cruzan entre sí.

\section{Reflexiones finales}

Las políticas que suelen recomendarse para mejorar el funcionamiento del mercado de trabajo de los jóvenes son, fundamentalmente, de dos tipos: formación y flexibilidad en salarios, contratos y dedicación. Aunque el lado de la demanda de trabajo no está presente en los análisis empíricos, en todos ellos se recuerda insistentemente el lado de la oferta en el mercado de trabajo no es la única dimensión a estudiar; es más, se apunta que no resulta creíble la idea de que actuando sólo por el lado de la oferta se pueda resolver el problema del desempleo juvenil y la escasa calidad de la transición del sistema educativo al mercado de trabajo en España.

Dos de las preguntas que seguimos haciéndonos hoy en día acerca del funcionamiento del mercado de trabajo de los jóvenes son por qué los jóvenes tienen tasas de paro más elevadas que la media y por qué las condiciones laborales de los jóvenes son peores que las de los adultos. Luis Toharia contribuyó a través de sus trabajos a la reflexión sobre estas preguntas, y en particular quisiéramos destacar aquí dos reflexiones concretas: la primera es acerca de los salarios de los jóvenes, y la segunda acerca de las condiciones laborales.

Una de las preguntas que subyace al hablar del salario de los jóvenes y que todos nos hacemos es si los salarios son una "barrera de entrada" al empleo para los jóvenes. Esta es una pregunta "trampa" ya que la barrera puede radicar tanto en que el salario sea muy bajo como en que sea muy alto, pero cuando se hace la pregunta siempre se está pensando en que es muy alto. Si se afirma que lo es, entonces se lanzará el mensaje de que los jóvenes tienen salarios elevados y que deben bajar para que mejoren sus tasas de ocupación. En el caso de la juventud en España, 
Toharia encuentra (ya en los años ochenta del siglo XX) que aplicar el análisis tradicional de que al bajar los salarios se reducirá el desempleo no parece apropiado por el siguiente argumento: los jóvenes españoles han aumentado considerablemente su nivel educativo (y no hay acuerdo todavía sobre si dicho incremento es insuficiente) y esto ha aumentado a su vez su salario de reserva. A esto se une la fuerte estructura familiar que actúa como "colchón" del desempleo, lo que hace que los jóvenes estén durante un periodo largo de tiempo buscando empleos esporádicos que les permitan correr con ciertos gastos personales pero realmente no ven el trabajo como un proyecto de vida. Si los salarios bajan este patrón se podrá incluso extender. Una forma de suavizar el impacto de los bajos salarios de los jóvenes es intervenir en el mercado de la vivienda, cuestión colateral sólo en apariencia. Pensemos que puede haber dos barreras de entrada al mercado de trabajo (para los jóvenes en especial): la primera es una formación que no se ajusta a lo que se demanda por parte de la empresa; y la segunda, una resistencia a la movilidad geográfica, que puede ocasionar problemas de ajuste. Una de las razones de la baja movilidad es el alto precio de la vivienda. Si abaratamos la vivienda (por las vías adecuadas y sin olvidar que la cultura española es la de ser propietario, lo que hace todo más complejo) resolveremos una vía de rigidez para la entrada. Si no se resuelve el tema de la movilidad, los esfuerzos por ajustar la formación pueden llegar a ser baldíos.

En cuanto a las condiciones laborales, entre las respuestas que se pueden plantear desde los trabajos de Luis Toharia a la cuestión de la calidad del empleo de los jóvenes españoles hay argumentos que apuntan a condiciones de tipo estructural: decir que la tasa de temporalidad de los jóvenes es la más alta de la Organización para la Cooperación y el Desarrollo Económica (OCDE) o de la Unión Europea no es una sorpresa; la sorpresa sería si España tuviese una tasa de temporalidad media más alta que la de los países de su entorno y los jóvenes la tuvieran más baja. Si vamos a hablar de empleo "atípico" (tiempo parcial, autónomos, temporales, combinación de trabajo y estudio) comparemos empleos "atípicos". Esa comparación internacional, por sí sola, ya sería más informativa.

Los jóvenes probablemente sean uno de los colectivos más indefensos (no pueden utilizar la experiencia laboral como "señal" de productividad y empleabilidad en el mercado, y además la falta de experiencia restringe sus derechos laborales) y es posible que en ellos se estén cebando los dos problemas fundamentales de la economía española: la baja productividad y la falta de competitividad. Flexibilizar el mercado de trabajo no parece resolver el primero; más bien ocurre al contrario. El segundo podría resolverse de dos formas: a) siendo más baratos (aquí cabe 
flexibilizar para reducir costes) y b) siendo "distintos". Para ser "distintos" debemos especializarnos, lo que requiere de una fuerte implicación del sistema productivo, mejorando tecnologías que permitan aprovechar el potencial capital humano que tiene la población española y muy especialmente la juventud. En este sentido parece que hay dos puntos de vista: en el primero se afirma que los jóvenes no tienen la formación que necesita el sistema productivo, por lo que habría que flexibilizar la entrada vía salarios y contratación; en el segundo se argumenta que los jóvenes han realizado una inversión educativa mientras que el sistema productivo sigue "obsoleto", no se ha modernizado y no necesita tanta formación como la que tiene nuestra mano de obra joven. La mejora de la tecnología es la "barita mágica" que ayudaría a mejorar la productividad y la competitividad. Para que la productividad del trabajo aumente, no sólo necesita formación, también necesita el capital físico adecuado.

La reflexión anterior apunta a las discusiones sobre el desajuste entre oferta y demanda de trabajadores jóvenes en términos de la formación que tienen. Pero también hemos recogido en párrafos anteriores que sería importante dotar a la mano de obra joven de mayor movilidad geográfica, lo que podría ayudar a mejorar los "emparejamientos" (ajustes) entre oferta y demanda, jóvenes y vacantes. Es imprescindible abordar este problema conjuntamente con el del acceso a la vivienda por parte de los jóvenes.

\section{Bibliografía}

Albert C., J.P. Juárez, R. Sánchez y Toharia, L. (1997) La transición de los jóvenes de la escuela al mercado de trabajo: un análisis longitudinal, Documentos de Trabajo del IVIE.

Albert C., J.P. Juárez, R. Sánchez y Toharia L. (2000) La transición de la escuela al mercado de trabajo: años noventa, Papeles de Economía Española, 86, 42-58.

Albert C. y Toharia L. (2000) El abandono o la persistencia en los estudios universitarios», Papeles de Economía Española, 86, 192-212.

Albert C., Davia M. A., Hernanz V. y Toharia L. (2002) Choosing between education, training and labour market entry", en K. Schömann y P. O'Connell, eds., Education, Training and Employment Dynamics: Transitional Labour Markets in the European Union, Cheltenham, Edward Elgar, 41-70. 
Albert C., J.P. Juárez, R. Sánchez y Toharia L. (2003) Del sistema educativo al mercado de trabajo: un análisis de flujos, Revista de Educación, 330, 137-155.

Albert C., Davia M. A., y Toharia L. (2002) The Process of Finding the First "Good" Job: The Case of Spain, en Economía de la Educación: Actas de las XII Jornadas de la AEDE, Editores: M. Jesús San Segundo, Rafael Zorrilla y Universidad Carlos III de Madrid, 251-261.

Albert C., Davia M. A., y Toharia L. (2007) Finding the first 'significant' job in Italy and Spain, en Lorenz Lassnigg, Helen Burzlaff, María A. Davia y Morten Larssen, eds. Lifelong learning: building bridges through transitional labour markets, Apeldoorn, Het Spinhuis, 23-43.

Albert C., García Serrano C. y Toharia L. (2007) "Los registros administrativos y los estudios de inserción laboral de los titulados universitarios: el caso de los titulados en Economía y ADE de la Universidad de Alcalá", en C. Marcos García, El papel de los registros administrativos en el análisis social y económico y el desarrollo del sistema estadístico, Madrid, Instituto de Estudios Fiscales, 291-302.

Albert C., Davia M. A., y Toharia L. (2008) To Find or not to Find a Significant Job: The Case of Spain, Revista de Economía Aplicada, 46 (16), 37-59.

Albert c., García Serrano C. y Toharia L. (2008) La inserción laboral de los universitarios: un estudio piloto de los licenciados en Economía y Administración y Dirección de Empresas de la Universidad de Alcalá. Universidad de Alcalá, Monografías Economía y Empresa (03). ISBN: 97884-8138-766-7.

Albert C., Davia M. A., y Toharia L. (2008) Exclusión social y pobreza: transición educativo - formativa e inserción laboral en la población joven Informe. Informe FIPROS. Disponible en (último acceso, 18 de julio de 2012): social.es/prdi00/groups/public/documents/binario/115788.pdf

http://www.seg-

Albert C., Davia M. A., y Toharia L. (2009) La transición de la educación secundaria (obligatoria y no obligatoria) al mercado laboral, Papeles de Economía Española, 119, 156-171. 
Cebrián I. y Toharia L. (2008) La entrada en el mercado de trabajo español: un análisis basado en la Muestra Continua de Vidas Laborales», Revista de Economía Aplicada, E-1 (vol. XVI), 137-172.

Muro J. y Toharia L. (1989) ¿Es elevado el salario de los jóvenes?, Revista de Economía y Sociología del Trabajo, n ${ }^{\circ}$ 1, 2-52.

Toharia L., Davia M. A., Hernanz V. (2001) Flexibilidad, juventud y trayectorias laborales en el mercado de trabajo español, Madrid, Centro de Investigaciones Sociológicas, Colección Opiniones y Actitudes nº 40, 2001.

Toharia L., Albert C. y Villacorba (2008) Estudio de la Situación Laboral de las personas egresadas en enseñanzas universitarias y de Formación Profesional reglada en Andalucía: año académico 2005-2006,. Servicio Andaluz de empleo, Consejería de Empleo, Observatorio ARGOS. 